\title{
The influence of in vitro fitness defects on pneumococcal ability to colonize and to cause invasive disease Jenny Fernebro ${ }^{1,2}$, Christel Blomberg ${ }^{1,2}$, Eva Morfeldt ${ }^{1}$, Hans Wolf-Watz ${ }^{3}$, Staffan Normark ${ }^{1,2}$ and Birgitta Henriques Normark*1,2
}

\author{
Address: ${ }^{1}$ Swedish Institute for Infectious Disease Control, 17182 Solna, Sweden, ${ }^{2}$ Dep. of Microbiology, Tumor and Cell Biology, Karolinska \\ Institutet, 17177 Stockholm, Sweden and 3Dep. of Molecular Biology, Umeå University, 90187 Umeå, Sweden \\ Email: Jenny Fernebro - jenny.fernebro@smi.ki.se; Christel Blomberg - christel.blomberg@smi.ki.se; Eva Morfeldt - eva.morfeldt@smi.ki.se \\ Hans Wolf-Watz - hans.wolf-watz@molbiol.umu.se; Staffan Normark - staffan.normark@smi.ki.se; \\ Birgitta Henriques Normark* - birgitta.henriques@smi.ki.se \\ * Corresponding author
}

Published: 18 April 2008

BMC Microbiology 2008, 8:65 doi:10.1/86/|47|-2/80-8-65

This article is available from: http://www.biomedcentral.com/I47|-2/80/8/65

(C) 2008 Fernebro et al; licensee BioMed Central Ltd.

This is an Open Access article distributed under the terms of the Creative Commons Attribution License (http://creativecommons.org/licenses/by/2.0), which permits unrestricted use, distribution, and reproduction in any medium, provided the original work is properly cited.
Received: 30 November 2007

Accepted: 18 April 2008

\begin{abstract}
Background: Streptococcus pneumoniae is a genetically diverse major human pathogen, yet a common colonizer of the nasopharynx. Here we analyzed the influence of defects affecting in vitro growth rate, on the ability of $S$. pneumoniae to colonize and to cause invasive disease in vivo.

Results: Of eleven different clinical isolates one serotype 14 carrier isolate showed a significantly longer generation time as compared to other isolates, and was severely attenuated in mice. To directly investigate the impact of growth rate on virulence, a panel of mutants in five non-essential housekeeping genes was constructed in the virulent TIGR4 background by insertion-deletion mutagenesis. Three of these mutants (ychF, hemK and yebC) were, to different degrees, growth defective, and showed a reduced invasiveness in an intranasal murine challenge model that correlated to their in vitro growth rate, but remained capable of colonizing the upper airways. The growth defect, as well as virulence defect of the hemK insertion-deletion mutant, was mediated by polarity effects on the downstream yrdC gene, encoding a probable chaperone in ribosome assembly.
\end{abstract}

Conclusion: We conclude that large fitness defects are needed to completely prevent pneumococci from causing invasive disease after intranasal challenge. However, even severe growth defects still allow pneumococci to persistently colonize the upper airways.

\section{Background}

Streptococcus pneumoniae is one of the major pathogens infecting humans worldwide. It is the most common cause of community-acquired bacterial pneumonia and childhood ear infections, but can also give rise to severe cases of meningitis and sepsis. It is estimated that 1-2 million people die from pneumococcal diseases every year [1].
The main pneumococcal virulence factor is the antiphagocytic capsular polysaccharide, depending on which pneumococci can be divided into at least 91 serotypes [2]. Other virulence factors described include cell wall components such as peptidoglycan, teichoic acid, lipoteichoic acid as well as cell surface proteins such as the cholinebinding proteins $[3,4]$ and the recently described pneumococcal pilus [5]. Signature-tagged mutagenesis (STM) 
has revealed a surprisingly large number of additional loci required for pneumococcal virulence in mice. Even though mutants in genes identified by STM are able to grow in vitro, it is not known to what extent these mutations cause fitness defects that might have an effect on virulence [6-8].

Despite causing severe diseases, pneumococci are also frequent colonizers of healthy individuals. $60-70 \%$ of children attending day-care centers may harbor these bacteria in their nasopharynx [9]. It is not fully understood which bacterial and host factors that contribute to the transition from carriage to disease. Invasive disease potential has been shown to differ between serotypes $[10,11]$, but other genetic factors are also important for disease outcome in humans $[10,12,13]$. Previously we have shown in animal models that not only serotype, but also clonal type (based on multi locus sequence typing, MLST and pulsed-field gel electrophoresis, PFGE), affects clinical outcome [12].

Whether or not variation in growth rate among pneumococcal isolates affects virulence has not been investigated. Here we monitored the in vitro growth rate for a series of clinical pneumococcal isolates with different ability to grow in blood after intraperitoneal challenge of mice [12]. These isolates belong to different serotypes and different clonal types as determined by MLST. To further evaluate the impact of in vitro fitness on virulence, we generated mutants defective in non-essential genes belonging to the pneumococcal core genome and known to be universally present in both pathogenic and non-pathogenic bacteria
[14] and therefore unlikely to represent bona fide virulence genes. We find that bacterial fitness defects, as monitored by bacterial growth rates in vitro, proportionally decreases pneumococcal virulence in animal models, without abolishing the ability to colonize the upper airways.

\section{Results \\ In vitro growth rates of pneumococcal isolates and the relationship to colonization and virulence in mice}

Previously, we studied a collection of clinical pneumococcal isolates of serotypes 1, 4, 6B, 7F, 14 and $19 \mathrm{~F}$ belonging to different clonal types [12]. After intranasal challenge only TIGR4 and isolates of type 6B caused invasive disease. However, after intraperitoneal challenge both the median survival time and end-point survival rate differed between the isolates. TIGR4 and the two isolates of types $6 \mathrm{~B}, 7 \mathrm{~F}$ and $19 \mathrm{~F}$ respectively were highly virulent after intraperitoneal infection, while isolates $1-\mathrm{B}, 14-\mathrm{A}, 1-\mathrm{A}$ and 14-B were less virulent in decreasing order (Table 1) [12]. More than $90 \%$ of the mice survived $>216$ hours when inoculated with 14 -B as compared to $50 \%$ with a challenge of 14-A.

To investigate whether the differences observed in survival in vivo could be correlated to differences in fitness, growth rate estimations and growth curves were made using a Bioscreen equipment (Figure 1A and 1B). All except one isolate showed generation times, in semisynthetic $\mathrm{c}+\mathrm{y}$ medium, between 33 and 37 minutes. These differences in growth rates did not fully correspond to the abilities of the

Table I: Bacterial strains used in this study

\begin{tabular}{|c|c|c|}
\hline Pneumococcal strains & Description & Source or reference \\
\hline \multicolumn{3}{|l|}{ Clinical isolates } \\
\hline I-A (BHN3I) & Serotype I invasive isolate of ST306 & [12] \\
\hline I-B (BHN32) & Serotype I invasive isolate of ST228 & {$[12]$} \\
\hline 6B-A (BHN49) & Serotype 6B invasive isolate of STI 38 & {$[12]$} \\
\hline 6B-B (BHN50) & Serotype 6B carrier isolate of STI 76 & {$[12]$} \\
\hline 7F-A (BHN55) & Serotype 7F invasive isolate of STI9I & {$[12]$} \\
\hline 7F-B (BHN56) & Serotype 7F carrier isolate of STI9I & {$[12]$} \\
\hline I4-A (BHN78) & Serotype 14 invasive isolate of ST I 24 & {$[12]$} \\
\hline I4-B (BHN83) & Serotype 14 carrier isolate of ST555 & {$[12]$} \\
\hline I9F-A (BHNI00) & Serotype I9F carrier isolate of STI62 & {$[12]$} \\
\hline I9F-B (BHN97) & Serotype I9F carrier isolate of ST425 & {$[12]$} \\
\hline \multicolumn{3}{|l|}{ Laboratory strains } \\
\hline TIGR4 (BHNI26) & Wild-type strain of serotype $4\left(\mathrm{Em}^{\mathrm{S}} \mathrm{Sm}^{\mathrm{S}}\right)$ & [16] \\
\hline TIGR4S (BHNI60) & TIGR4 spontaneous streptomycin resistant mutant $\left(\mathrm{Sm}^{\mathrm{R}}\right)$ & This study \\
\hline TIGR4 $\Delta y c h F(B H N I 68)$ & $y c h F(S P 0004):: e r m\left(\mathrm{Em}^{\mathrm{R}}\right)$ & This study \\
\hline TIGR4 $\Delta r l u D(B H N I 69)$ & rluD (SP0929)::erm (EmR) & This study \\
\hline TIGR4Asmf (BHNI70) & $\operatorname{smf}(\mathrm{SPI}$ 266)::erm (EmR) & This study \\
\hline TIGR4AhemK (BHNI7I) & hemK (SPI02I)::erm $\left(\mathrm{Em}^{\mathrm{R}}\right)$ & This study \\
\hline TIGR4 $\Delta y e b C$ (BHNI72) & yebC (SPI922)::erm (EmR) & This study \\
\hline TIGR4ShemK- (BHNI73) & Point mutations in hemK (SPI02I) $\left(\mathrm{Sm}^{\mathrm{R}}\right)$ & This study \\
\hline TIGR4SyrdC- (BHNI74) & Point mutations in yrdC (SPI022) $\left(\mathrm{Sm}^{\mathrm{R}}\right)$ & This study \\
\hline TIGR4SSPI023- (BHNI75) & Point mutations in SPIO23 (SmR) & This study \\
\hline
\end{tabular}




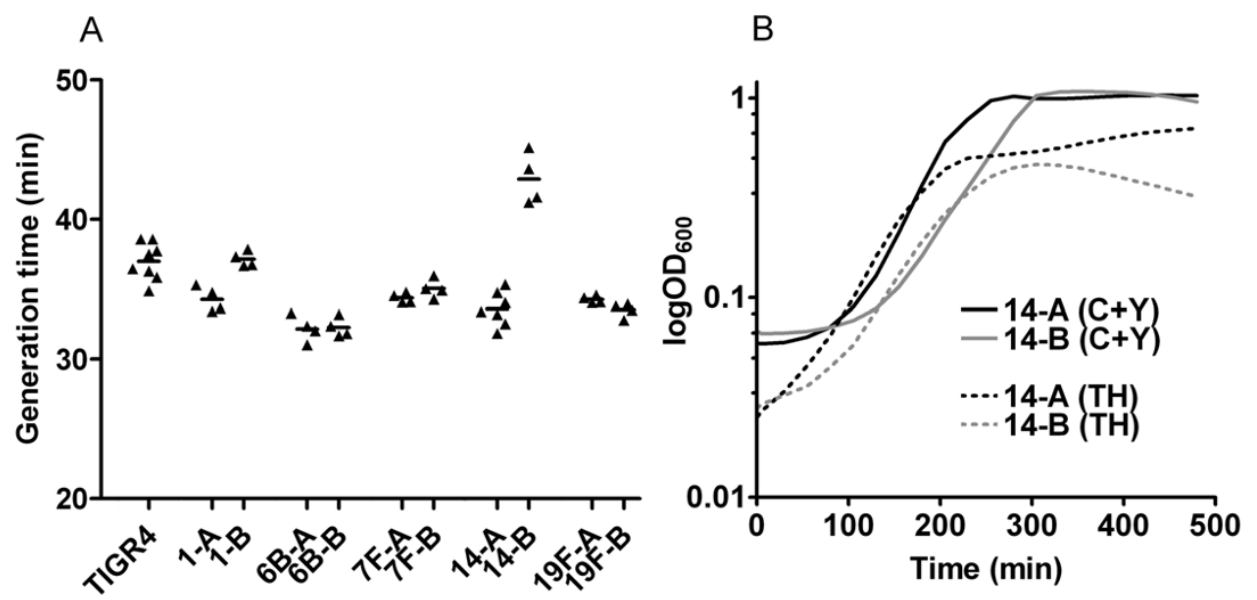

Figure I

A) Generation times in c+y of clinical isolates of serotypes I (I-A and I-B), 4 (TIGR4), 6B (6B-A and 6B-B), 7F (7F-A and 7F-B), I 4 (I 4-A and I4-B) and I9F (I 9F-A and I9F-B).B) Growth curves of the two type I4 clinical isolates in $\mathrm{C}+\mathrm{y}$ medium and $\mathrm{TH}$ medium.

same isolates to grow in blood in vivo and to cause systemic disease [12]. Thus, isolate 1-B was more virulent than 1-A despite that the former isolate showed a slower in vitro growth rate. Nevertheless, it is interesting to note that the two $6 \mathrm{~B}$ isolates showing the highest growth rates in vitro were the most virulent ones after intraperitoneal infection in mice. Only the Type14-B carrier isolate exhibited a significantly longer generation time (43 minutes) compared to all other isolates, which was in line with its low virulence potential in mice.

Comparative genomic hybridizations were next carried out on the isolates 14-A and 14-B to identify gene content differences that could explain the fitness defect of the latter isolate. Microarrays were used, representing the full genomes of strains R6 [15] and TIGR4 [16]. The invasive serotype 14 isolate of ST124 (14-A) and the carrier isolate

Table 2: Microarray analysis of the clinical isolates I4-A and I4-B with respect to genes identified in STM [6-8]. Gene annotations are given according to TIGR4 [16].

\begin{tabular}{|c|c|c|c|c|}
\hline Gene & Description & Ref & I4-A STI 24 & I4-B ST555 \\
\hline SP 007I & immunoglobulin AI protease & {$[6,8]$} & - & + \\
\hline SP 0143 & Hypothetical protein & {$[8]$} & - & + \\
\hline SP 0265 & bgla; 6 phospho beta glucosidase & [6] & - & + \\
\hline SP 0396 & mtlf; Mannitol specific enzyme IIA component & [6] & - & + \\
\hline SP 0474 & pts eii; Phosphotransferase system sugar specific Ell component & [6] & - & + \\
\hline SP 1321 & $v$ type sodium ATP synthase subunit $K$ & [6] & - & + \\
\hline SP 1328 & sodium:solute symporter family protein & [6] & - & + \\
\hline SP 0268 & alkaline amylopullulanase putative & {$[6]$} & + TIGR4 & $+R 6$ \\
\hline SP 0629 & conserved hypothetical protein & [7] & + TIGR4 & $+\mathrm{R} 6$ \\
\hline SP 0933 & pyrroline 5 carboxylate reductase & [7] & + TIGR4 & $+R 6$ \\
\hline SP 1018 & tdk; Thymidine kinase & [6] & + TIGR4 & $+R 6$ \\
\hline SP 1029 & RNA methyltransferase TrmA family & [6] & + TIGR4 & $+R 6$ \\
\hline SP 1343 & ptrb; Protease II (oligopeptidase B) & {$[6]$} & + & - \\
\hline SP I344 & Conserved hypothetical protein & [6] & + & - \\
\hline SP 1433 & Hypothetical protein & {$[6]$} & + & - \\
\hline SP 1434 & $A B C$ transporter ATP binding/permease protein & [6] & + & - \\
\hline SP 2095 & Conserved hypothetical protein & [6] & + & - \\
\hline SP 2164 & pts eii; Phosphotransferase system sugar specific Ell component & [6] & + & - \\
\hline
\end{tabular}

Note: Genes that are present are designated +, genes that are absent are designated -. In cases where sequence differences were found between the test isolates, subscripts indicate which reference strain the test strain hybridized to. 
of ST555 (14-B) differed from one another at approximately 196 genes including some variable regions. R6specific cluster 4 [17], C11* [17], C13* [17] and most of TIGR4-specific cluster 7 [17], also known as PPI-1, were present in 14-A but absent in 14-B. TIGR4-specific cluster 1 and 9 as well as $\mathrm{C} 4 *$, C5* and part of R6-specific cluster 1 [17] were absent in 14-A, but present in 14-B. In addition, the two isolates differed from each other at 18 loci found to affect virulence by signature-tagged mutagenesis screen (Table 2). Six of these loci were absent in 14-B, seven were absent in 14-A and five had differences in sequence between the two isolates. One of the genes that varied in sequence was SP1018, which is included in the same operon as hemK (see below). Also, SP1019 of the hemK operon was missing in the slow-growing 14-B isolate, where SP1018 was directly followed by SP1020. SP1019 has previously been reported to be missing from some sequenced clinical isolates (see NCBI url in Availability and requirements section) [18].

Even though we found large differences in the content of virulence and putative metabolic genes between the two type 14 isolates, we could not pinpoint one specific gene responsible for the observed defect in growth and virulence. Therefore we next studied isogenic mutants in conserved housekeeping genes in the pneumococcal genome.

\section{In vivo studies of isogenic mutants with different generation times}

To investigate the association between in vitro growth rate and in vivo ability to colonize and cause systemic disease insertion-deletion mutants were created in five genes in TIGR4, one of the most virulent strains in C57BL/6 mice in our collection. Microarray analysis of 40 pneumococcal isolates belonging to different serotypes and clones revealed that all carried the five genes, indicating that they belong to the pneumococcal core genome (data not shown). These highly conserved genes are present in all pathogenic as well as non-pathogenic bacterial species investigated so far [14], hence it is unlikely that they represent bona fide virulence genes. They were chosen since they appear to be house-keeping genes and gene inactivation would likely lead to fitness defects. They all have homologues in E. coli, hence we used their E. coli designations and named the mutants TIGR4 $\Delta y c h F$ (SP0004), TIGR4 $\Delta r l u D$ (SP0929), TIGR4 $\Delta s m f$ (SP1266),

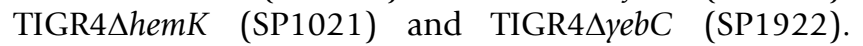
When in vitro growth rates were measured in c+y semisynthetic medium, the rluD and smf mutants showed almost wild-type fitness with generation times of 39 and 36 minutes respectively, as compared to 37 minutes for the wildtype TIGR4 strain ( $\mathrm{p}=0.0596$ and $\mathrm{p}=0.4452$ ) (Figure $2 \mathrm{~A}$ and $2 \mathrm{~B})$. However, the insertion-deletion mutants of $y \mathrm{chF}$, hemK and yebC all had reduced growth rates, 42, 65 and 48 minutes respectively $(\mathrm{p}=0.0003, \mathrm{p}<0.0001$ and $\mathrm{p}<$
$0.0001)$. The growth defect was especially pronounced for the hemK mutant, showing smaller colonies on blood agar plates. The growth differences for the mutant collection were also recorded in $\mathrm{TH}$ medium (Figure $2 \mathrm{C}$ ). Also in this medium the hemK mutant showed the most pronounced growth rate defect and reached a lower density at stationary phase.

To mimic the natural route of infection, C57/Bl6 mice were challenged intranasally with the five insertion-deletion mutants respectively. All mutants gave rise to the same type of disease, but with different frequencies. For those mice where bacteria could be cultured from blood, health status rapidly and irreversibly deteriorated. At the time of sacrifice all mice with invasive pneumococcal infection had similar amounts of bacteria in the blood and lungs $\left(5 \times 10^{7} \mathrm{CFU} / \mathrm{ml}\right.$ and $1 \times 10^{5} \mathrm{CFU} / \mathrm{mg}$ respectively), irrespective of challenge strain. Thus, once bacteremia occurred, even the severely growth defective hemK mutant was not cleared from the blood stream. Three of the five mutants, TIGR $4 \Delta y c h F$, TIGR $4 \Delta$ hemK and TIGR4 $\Delta y e b C$, gave a higher end-point survival than the wild-type TIGR4 strain with $55 \%\left(\chi^{2}=10.25 ; \mathrm{p}=0.0014\right)$, $77 \%\left(\chi^{2}=18.41 ; \mathrm{p}<0.0001\right)$ and 56\% $\left(\chi^{2}=7.369 ; \mathrm{p}=\right.$ 0.0066 ) survival respectively by 192 hours, compared to $31 \%$ for TIGR4 (Figure 2D). In contrast, TIGR $4 \Delta r l u D$, and TIGR4 $\Delta s m f$, were not attenuated, with survival rates of $38 \%\left(\chi^{2}=0.3529 ; \mathrm{p}=0.5525\right)$ and $32 \%\left(\chi^{2}=0.0002264\right.$; $\mathrm{p}=0.9880)$ respectively. There was a good relationship between end-point survival and in vitro growth rate (Figure $2 \mathrm{E}$ ). The growth-defective mutants were capable of colonizing the mice to a similar extent 8 days post infection as TIGR4 (Figure 2F).

Out of the five mutants, the growth defect was most pronounced for the hemK mutant. Since insertion-deletion mutagenesis is likely to give polar effects on downstream genes in the same operon, in-frame mutants were constructed in hemK and in the two genes directly downstream of hemK (SP1022 and SP1023) in order to pinpoint the responsible gene for the growth defect (Figure 3). Nucleotides were altered in the 5 '-end of the open reading frames, introducing stop codons. The slow-growing insertion-deletion mutant of hemK gave smaller colonies than TIGR4 on blood agar plates. Of the in-frame mutants only the mutant in SP1022 (TIGR4SyrdC-) gave the same phenotype with small colonies, while the inframe mutants of hemK (TIGR4ShemK-) and SP1023 (TIGR4SSP1023-) both showed wild-type colony size. The reduced colony size for TIGR4SSP1022- corresponded to a reduced number of bacteria/colony $\left(6 \times 10^{5}\right.$ as compared to $2 \times 10^{6}$ for TIGR4S) after 20 hours of incubation on blood agar plates. Growth rate estimations with Bioscreen verified that only TIGR4SyrdC- gave a longer generation time in $\mathrm{c}+\mathrm{y}$ medium than TIGR4S, 65 minutes as com- 

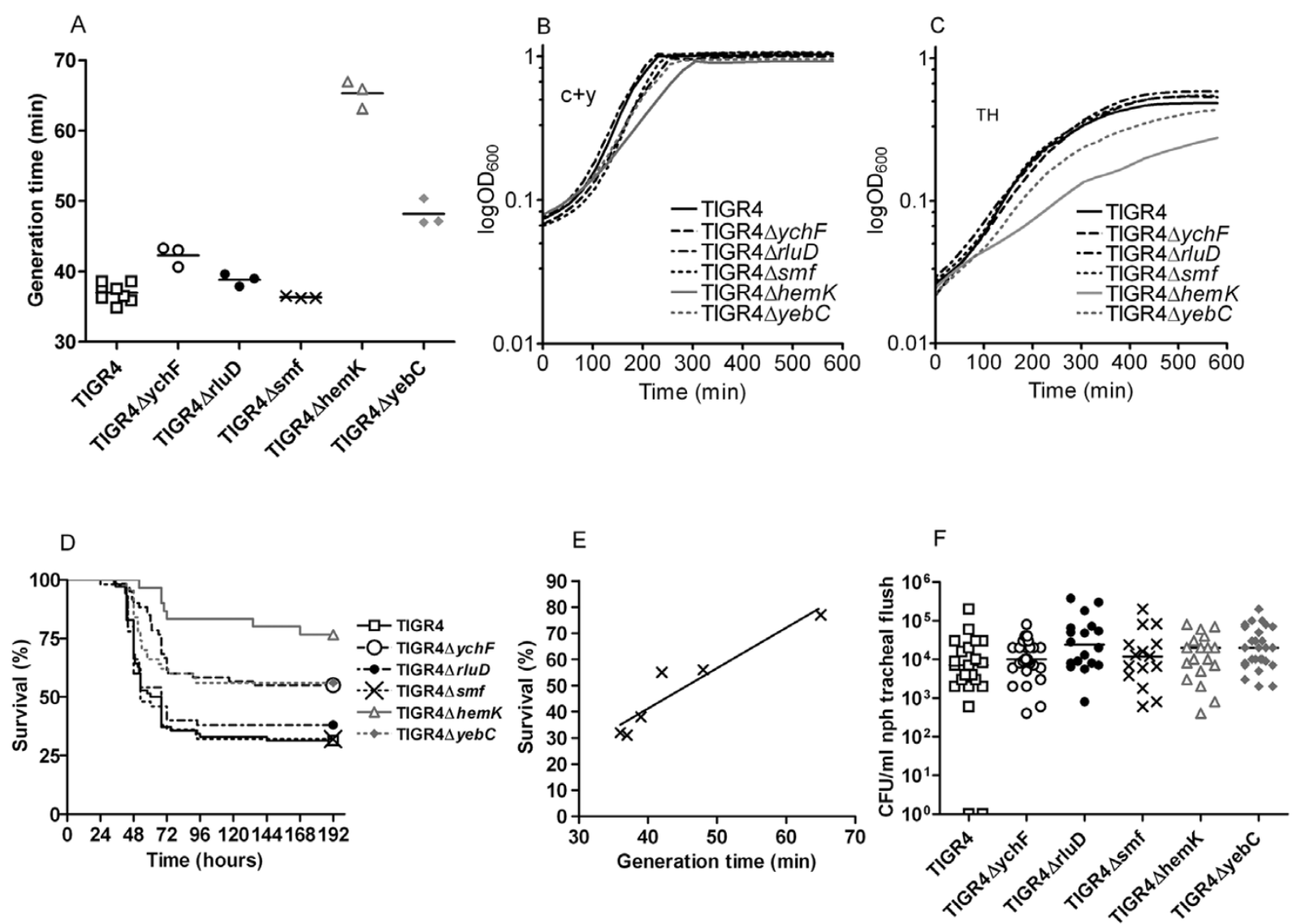

Figure 2

Experiments with insertion-deletion mutants in the TIGR4 background.A) Generation times in c+y medium. B) Growth curves in c+y medium. C) Growth curves in TH medium. D) Survival of mice after intranasal infection. E) Generation time of mutants versus survival of infected mice. F) Colonization of mice 8 days after intranasal infection.

pared to 37 minutes ( $\mathrm{p}=0.0005)$ (Figures $4 \mathrm{~A}$ and $4 \mathrm{~B})$. The generation times for TIGR4ShemK $K^{-}$and TIGR4SSP1023- were 39 minutes $(\mathrm{p}=0.0591)$ and 38 minutes $(\mathrm{p}=0.6780)$ respectively. The same relative differences in in vitro growth rate for the different in frame mutants was also found after growth in TH-medium, with TIGR4Shem $K^{-}$and TIGR4SSP1023-exhibiting similar growth rate as TIGR4S and TIGR4SyrdC- exhibiting a severe growth defect (Figure 4C).

Next, mice were challenged intranasally with TIGR4S, TIGR4Shem $K^{-}$or TIGR4SyrdC- (Figure 4D). The survival rates with TIGR4Shem $K^{-}$and TIGR4S were $40 \%\left(\chi^{2}=\right.$ $0.06550 ; \mathrm{p}=0.7980)$, with median survival time of $59 \mathrm{~h}$ and $68 \mathrm{~h}$ respectively. All mice survived after challenge with TIGR4SyrdC- $\left(\chi^{2}=18.03 ; \mathrm{p}<0.0001\right)$. However, 12 out of 14 mice infected with this mutant remained colonized, as determined by CFU count from nasopharynealtracheal lavage at day 8 post-infection (Figure 4E), albeit at a lower level than those challenged with TIGR4S (median of $2 \times 10^{3} \mathrm{CFU} / \mathrm{ml}$ lavage versus $6.8 \times 10^{4} \mathrm{CFU} /$ $\mathrm{ml}$ lavage $(\mathrm{p}=0.0151))$.

\section{Discussion}

The pneumococcal genome consists of a core genome representing about $70 \%$ of the total gene content. The remaining 30\% represents variable genes that may be present or absent in different isolates (unpublished data). Several of these variable genes are predicted to have metabolic functions, and therefore do not represent true virulence genes, even though in vivo screens using signaturetagged mutagenesis have identified a number of them as necessary to cause invasive disease in murine models [68]. Since in vitro fitness might be one explanation for differences found in vivo we aimed to determine whether in vitro fitness, as determined by in vitro growth rates, correlates to virulence in vivo. We used two clinical isolates each of serotypes $1,6 \mathrm{~B}, 7 \mathrm{~F}, 14$ and $19 \mathrm{~F}$ with known virulence in an intraperitoneal mouse model [12] and compared growth rates with a Bioscreen apparatus. The two isolates 


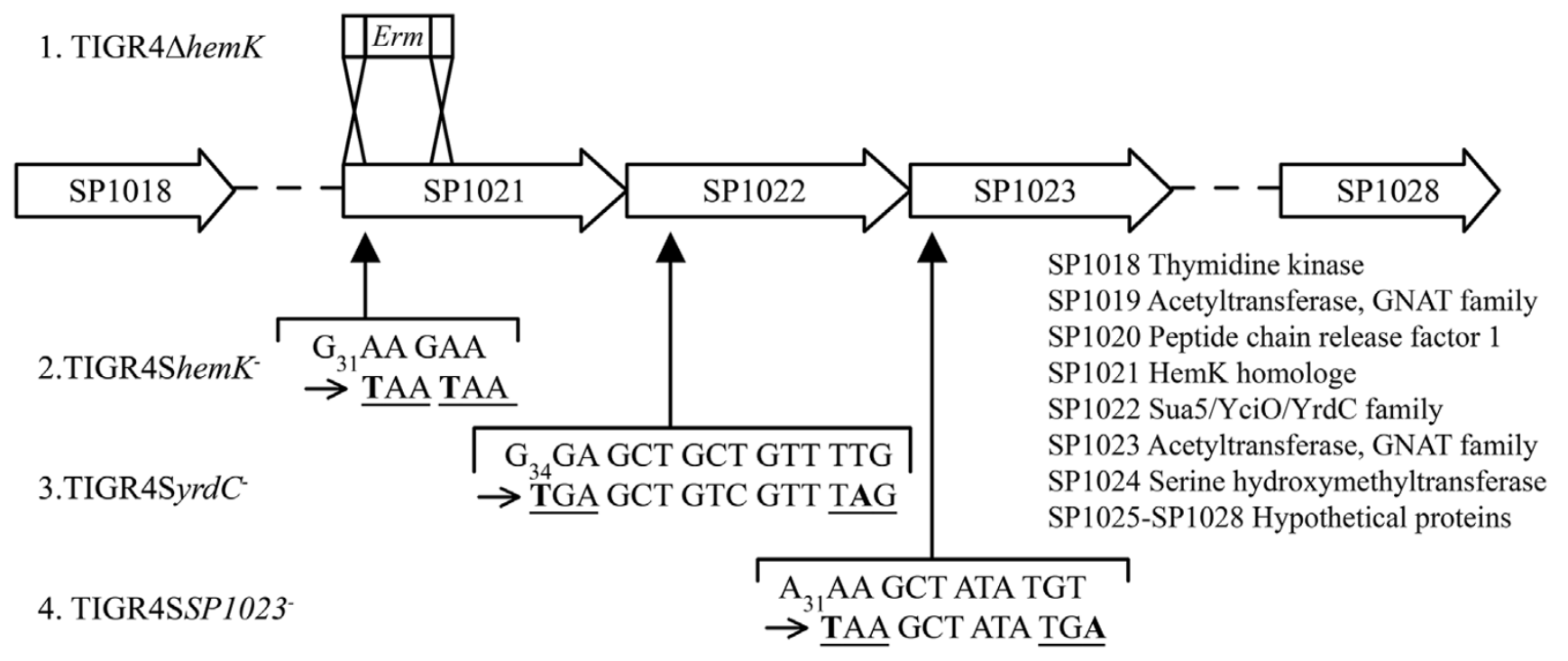

Figure 3

The hemK operon and the mutants created in it. TIGR4 $\Delta$ hemK was made by insertion-deletion mutagenesis with an erythromycin cassette, while the other mutants contain in-frame stop codons (underlined) in the very beginning of the target genes. Alterations are shown in bold and the subscripts indicate the nucleotide number of the first alteration in each gene.

of each serotype were chosen to be in most cases one carriage isolate and one invasive isolate that were genetically unrelated based on MLST profiles (Table 1). Differences in virulence had previously been detected between the two type 1 isolates, and an even more pronounced difference was found between the two type 14 isolates [12]. Major differences in in vitro growth rates between strains of the same serotype were detected only for type 14 isolates, suggesting in vitro growth to be an important parameter for the outcome in vivo. The two type 14 isolates were genetically unrelated by MLST (of ST124 and ST555 respectively) and microarray analysis, differing from each other at 18 loci found to effect virulence by STM screens (Table 2 ). Seven of these loci were absent in the more virulent 14A isolate, while six were absent in 14-B. In five of the loci there were differences in sequence between the two isolates, with 14-A carrying genes equal to TIGR4, and 14-B having the R6 equivalents. Of the genes missing in slowgrowing 14-B, at least two are predicted to be involved in transport functions (SP1434 and SP2164), but it is not known if defects in these genes affect growth.

For the two isolates of type 1, the differences in virulence could not be explained by differences in growth rates. Several features, besides growth fitness in a rich complex medium such as $\mathrm{c}+\mathrm{y}$, may influence the disease potential of a pneumococcal strain including the capsular type being expressed. This is also reflected by the fact that the differences seen in in vitro growth rate between the clinical isolates of different serotypes did not fully correspond to differences in mice virulence as monitored after intraperitoneal challenge. Ability to retrieve carbohydrates from complex glycoconjugates is likely important for pneumococcal ability to grow in different host environments [19]. Virulence-associated differences among clinical isolates in carbohydrate retrieval may only be revealed during in vitro growth using carbon sources mimicking the host environment, and was not further investigated in this paper. Instead we attempted to correlate the relationship between in vitro growth rate and in vivo virulence in mice creating a panel of mutants in the TIGR4 background deficient in highly conserved non-essential house keeping genes that were unlikely to affect carbohydrate metabolism of Streptococcus pneumoniae. TIGR4 was used since it is fully sequenced and since it is the most virulent strain in our collection after intranasal challenge.

The five candidate genes in the pneumococcal core genome were chosen based on a previous publication by Garbom et al. [14]. Garbom et al. showed that these genes are needed for full virulence of Yersinia in vivo, but they have only been considered as housekeeping genes in pneumococci. Their functions have been predicted either in E. coli or in other species, except for yebC. The E. coli YchF protein belongs to a group of 11 universally conserved GTPases [20]. Studies in Haemophilus influenzae have shown that YchF contains binding sites for both GTP and double-stranded nucleic acid, indicating that YchF may be part of a nucleoprotein complex and function as a GTP-dependant translation factor [21]. rluD encodes a 

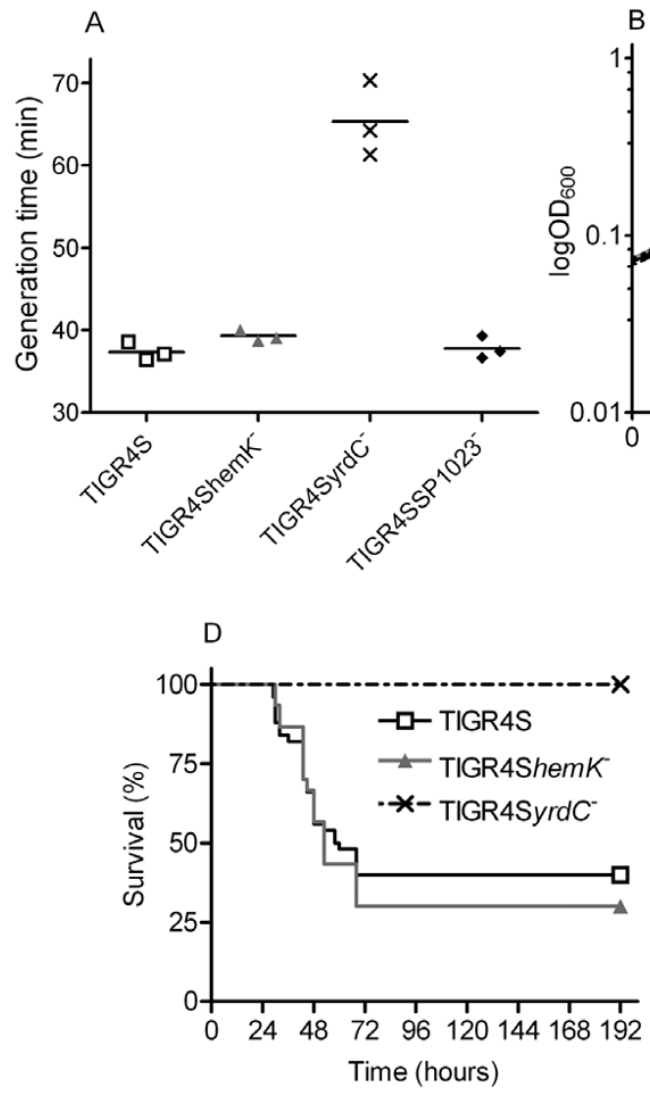
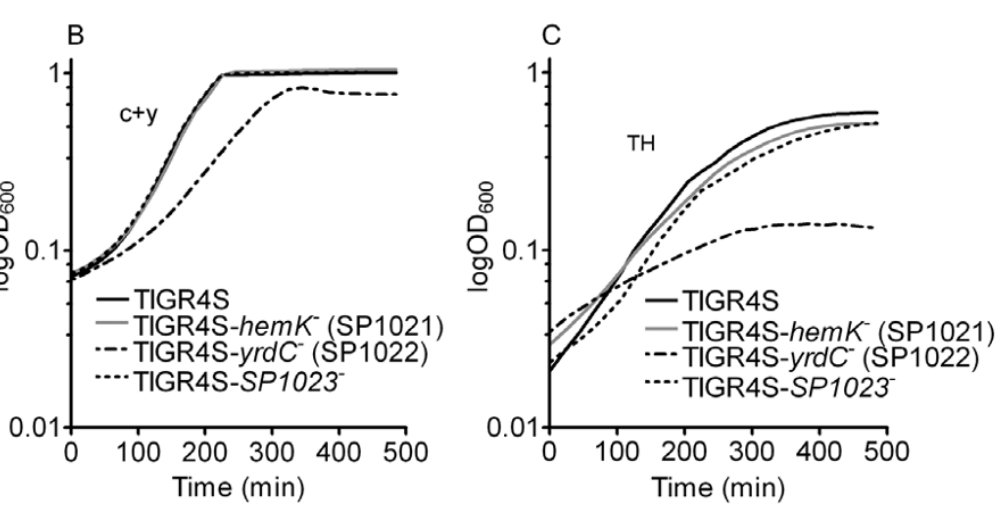

\section{$E$}

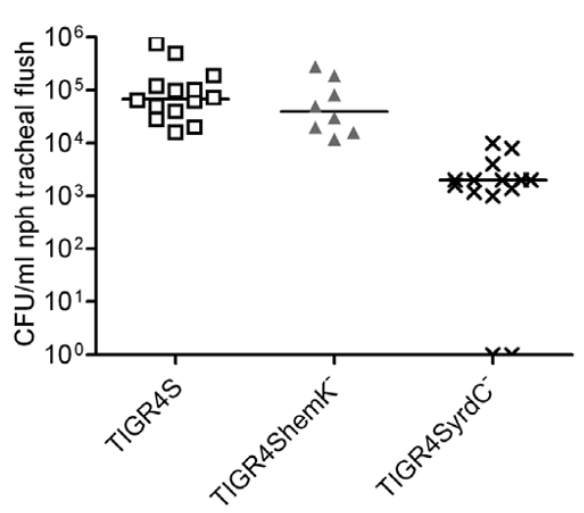

Figure 4

Experiments with in-frame mutants in the TIGR4S background.A) Generation times of strains grown in c+y medium. B) Growth curves in c+y medium. C) Growth curves in TH medium. D) Survival of mice after intranasal infection. E) Colonization of mice 8 days after intranasal infection.

pseudouridine synthase in E. coli which is responsible for the production of 23S rRNA pseudouridines 1911, 1915 and 1917 [22]. This protein is non-essential in E. coli, but affects growth [22]. SP0927, which is transcribed upstream of the pneumococcal homolog to rluD has been identified as essential for lung infection in mice in a signature mutagenesis screen (STM) [6]. smf has a homologue in H. pylori, B. subtilis, and Streptococcus pneumoniae, named $d p r A$ (SP1266), and is involved in natural competence [23-25]. It has only been linked to virulence in Yersinia and N. meningitidis $[14,26]$. The pneumococcal DprA protein is proposed to play a role in the late stage of transformation, by binding to incoming single stranded DNA and bringing it to the RecA recombinase. Finally, hemK in E. coli encodes an N(5)-glutamine methyltransferase that modifies peptide release factors [27].

Insertion-deletion mutants (potentially creating polar effects on transcriptionally downstream genes) were constructed for all five genes, $y c h F, r l u D, s m f$, hemK and $y e b C$ in the TIGR4 background. Defects in in vitro growth were observed for mutants in $\gamma c h F$, hemK and $y e b C$ respectively, where the HemK-deficient mutant was most severely attenuated with an $80 \%$ longer generation time as compared to the wild-type TIGR4. In an intranasal mouse model of infection, mutations that did not affect in vitro fitness, i.e. $r l u D$ and $s m f$, did not influence virulence, while all mutants with growth defects $(y c h F$, hemK and $y e b C$ ) were also attenuated in virulence. The reduction of virulence correlated to the extent of growth defect observed. Hence, the hemK mutant was most attenuated, but still capable of causing severe invasive disease in $27 \%$ of the animals. Long-term colonization of the upper airways was not affected by any of the mutants. Thus, fitness defects reducing growth rate in vitro did not affect nonsymptomatic carriage, but affected the likelihood of transition from carriage to invasive disease. However, once invasive disease occurred there was no apparent effect of a fitness defect on the progression of invasive disease. 
For the mutants in $y c h F$ (SP0004) and yebC (SP1922), we did not investigate possible effects on neighboring genes. The pneumococcal homologue to $y c h F$ was reported as potentially essential by Thanassi et al., but Song et al. showed that this effect was due to polarity $[28,29]$. Instead Song et al. identified SPR0003, the R6 equivalent of SP0003, which is probably transcribed together with $y c h F$, as essential. Therefore, it is possible that the phenotype of our $y c h F$ insertion-deletion mutant is due to effects on SP0003 or polar effects on another essential gene SP0005 [29] encoding a putative peptidyl-tRNA hydrolase. Also in the case of yebC (SP1922), interesting genes can be found in close proximity. About $1 \mathrm{~kb}$ downstream of yebC is ply, encoding the well-known pneumococcal virulence factor pneumolysin [30]. These genes are however not believed to be co-transcribed, since the ply gene is transcribed from its own promoter [31].

In S. pneumoniae, hemK is the fourth gene out of eleven in an operon that has previously been reported to be associated with virulence $[32,33]$. Two groups have reported the effects of mutations in hemK or the hemK operon in $S$. pneumoniae. While Marra et al. observed a significant effect on virulence, Orihuela et al. did not observe any attenuation [32,33]. Hava and Camilli identified SP1023 (two genes downstream of hemK) as essential for lung infection in mice using STM, but did not study the mutant further [6]. To investigate which gene(s) in this operon was attributed to the observed defect in growth and virulence, we constructed in-frame mutants for hemK and the two genes downstream of hemK, SP1022 and SP1023. The HemKdeficient insertion-deletion mutant showed smaller colonies on blood agar plates, hence we studied the in-frame mutants for this phenotype and found it only in the SP1022 mutant. This mutant also had a considerably longer generation time in $\mathrm{c}+\mathrm{y}$ as well as in $\mathrm{TH}$ medium compared to the parental strain, which was not the case for the other in-frame mutants. Also, the SP1022 mutant was completely non-virulent in vivo, while the non-polar hemK in frame mutant gave wild-type virulence. In this set of experiments all derivatives used carried a mutation in $r p s L$ giving streptomycin resistance. The lower virulence observed for TIGR4S as compared to TIGR4 is likely due to the fitness cost of this $r p s L$ mutation as previously shown for Salmonella typhimurium [34]. The complete attenuation of the in-frame mutation in SP1022 likely reflects the added fitness defect contributed by the $r p s L$ mutation. SP1022 is a homologue to $y r d C$ in E. coli, which was recently reported to be responsible for ribosome maturation [35] and mutations in this gene resulted in severe defects in growth. Non-ribosomal factors in ribosome subunit assembly such as YrdC are emerging targets for new antibacterial drugs [36]. We cannot rule out that also SP1023 (as well as other genes downstream in the operon) may be involved in virulence, without affecting in vitro fitness. SP1023 was picked up in an STM screen by Hava and Camilli [6]. The method used by Hava and Camilli to construct the mutant library may have lead to effects on the expression from neighboring genes within the operons of the targeted genes. Hence, it is possible that the phenotype reported for SP01023 is due to effects on yrdC (SP1022) expression.

\section{Conclusion}

Here we found support both among our clinical isolates studied as well as among the constructed mutants that in vitro growth affects invasiveness in vivo. However, all TIGR4 mutants with reduced growth rate remained capable of causing severe invasive disease in a fraction of mice, and were still able to colonize the upper airways to the same level as the wild-type strain. Also, the slowly growing ST555 isolate of serotype 14 (14-B) was isolated from the nasopharynx of a human being. Our results therefore suggest that pneumococci with metabolic or other fitness defects may prevail in the carrier population, and potentially also cause invasive disease. Such strains could possibly act as reservoir for the build up of better-fit strains that also are more capable of generating invasive disease. It is surprising that so many pneumococcal loci have been shown, by signature-tagged mutagenesis, to be required for virulence in mice [6-8]. Revisiting this collection of mutants, asking which ones also exhibit in vitro fitness defects, may significantly reduce the number of pneumococcal loci that are directly involved in overcoming host defenses during invasive disease. Furthermore, it may be fruitful to identify conserved surface-exposed proteins that provide a growth defect when inactivated and use these proteins as drug targets or as recently reported as vaccine targets [37].

\section{Methods \\ Bacterial strains used}

The bacterial strains used in this study are listed in Table 1. Bacteria were grown on blood agar plates over night, were resuspended in glucose medium supplemented with $10 \%$ horse serum and inoculated 1:10 in semisynthetic $\mathrm{c}+\mathrm{y}$ medium [38], and grown until appropriate $\mathrm{OD}_{620}$.

\section{In vitro fitness as determined by monitoring growth}

To determine fitness of clinical isolates and mutants, bacterial growth in semisynthetic $\mathrm{c}+\mathrm{y}$ medium [38] was monitored in Bioscreen (Labsystems, Finland). Measurements were made at $\mathrm{OD}_{600}$ every fifth minute for sixteen hours and the experiment was repeated three times. Growth curves were made and generation times were calculated from the slope of the growth curves at mid-log phase. Growth curves were also made based on growth in TH medium. 


\section{Microarray analysis}

Comparative genomic hybridizations were carried out on two type 14 clinical isolates using a reference design as previously described [39]. Four replicate experiments, including dye-swap, were performed. Oligonucleotides were based on predicted open reading frames of the two sequenced strains R6 [15] and TIGR4 [16]. The data were analyzed using Genepix pro 6.0 and the R Project for Statistical Computing (see Availability and requirements section for url) as previously described [39]. For statistical analysis we used a Bayesian linear model [40] and the Holm multiple testing correction to adjust individual pvalues. This method was used to compare the isolates to the reference strains. Genes were considered absent if they had a p-value less then 0.01 within $\mathrm{M}$-value less than -1 and present if they had an M-value of more than -0.8 . Genes were considered to have sequence differences when one isolate bound to the oligonucleotide corresponding to R6 and the other to the oligonucleotide corresponding to TIGR4.

\section{Housekeeping genes selected for further analysis}

To study the effect of growth on virulence, five universally conserved housekeeping genes shown to affect virulence in Yersinia [14] were selected. All five genes have homologues in E. coli, designated $\gamma c h F, r l u D, s m f$, hemK and yebC. The respective pneumococcal equivalents in the sequenced TIGR4 and R6 strains are SP0004/SPR0004, SP0929/SPR0830, SP1266/SPR1144 (dprA), SP1021/ SPR0925 and SP1922/SPR1738 respectively $[15,16]$.

\section{Construction of mutants using insertion-deletion mutagenesis}

Flanking regions of target genes were amplified from TIGR4 using specific primers (Appendix) giving either $A p a \mathrm{I}$ or BamHI restriction sites. The erythromycin cassette from pVA838 [41] was amplified with ApaI and BamHI termini (Appendix). All fragments were digested and ligations were performed with upstream, downstream and erythromycin fragments. TIGR4 was transformed with the ligation products, with selection for $\mathrm{Em}^{\mathrm{R}}$. Transformations were made by adding DNA and CSP-2 [42] to the recipient pneumococcal strain at $\mathrm{OD}_{620}=0.1$, followed by incubation on ice, at $31^{\circ} \mathrm{C}$ and finally at $37^{\circ} \mathrm{C}$. Transformants were verified by PCR with control primers (Appendix).

\section{Construction of stop codon mutants (Janus)}

Stop codon mutants were constructed for hemK and the two genes downstream of hemK (SP1022 and SP1023). These were produced using the Janus cassette [43] with both positive (kanamycin) and negative (streptomycin) selection. The wild-type strain was a streptomycin-resistant TIGR4 mutant, TIGR4S, spontaneously obtained through selection on streptomycin-containing agar plates and containing a substitution (K56 $\rightarrow$ R56) in the $r p s L$ gene, previously reported to cause streptomycin resistance [44]. A similar construct as for the insertion-deletion mutants was made, but with the Janus cassette instead of the erythromycin cassette, using primers DAM406 and DAM351 [43]. This ligation mix was first transformed into TIGR4 with selection on kanamycin-containing plates. PCR was run over the full construct and this fragment in turn was transformed into TIGR4S. Positive clones of TIGR4S were then transformed with PCR products containing stop codons. These were introduced about ten amino acids downstream of the initial ATG.

\section{In vivo studies}

5-8 weeks old C57BL/ 6 mice were inoculated intranasally with $5 \times 10^{6} \mathrm{CFU}$. Mice were monitored for 8 days to assess the health status by clinical scoring and blood samples taken daily. The mice health status was monitored according to the following scores: 0 = healthy, $1=$ piloerection, 2 = reduced motility, $3=$ more pronounced reduced motility, $4=1,2,3$ more pronounced and $5=$ moribund. Mice were sacrificed when they reached score $\geq 3$. Surviving mice were killed on day 8 and a nasopharyngealtracheal flush was performed to monitor colonization. All experiments were repeated at least three times. The studies were approved by The Ethical Committee for Animal Experiments in Stockholm. All animals were kept with a 12-hours light/dark cycle and had access to standard food and tap water ad libitum.

\section{Statistical analysis}

For survival studies, differences were analysed by the Kaplan-Meier analysis log-rank test. The generation times were analysed using the student $t$-test (two-tailed). $P<$ 0.05 was considered significant. 
Table 3: Primers used in this study

\begin{tabular}{|c|c|}
\hline Primer & Sequence \\
\hline YchF-up5' & AAGTACACAAGAGAGTCGTCCGAT \\
\hline YchF-up3' & TTGGGCCCTCTCCGTTTTTCATTTCAATCCC \\
\hline YchF-down5' & TTGGATCCAATGGTGTCAATTAGGTTGGAA \\
\hline YchF-down3' & TGATCATATTCTTCACCAACTGAGA \\
\hline YchF-Cl & GAAATTTCAGCAAGCACTCC \\
\hline YchF-C2 & ACTGATGGATAAACGCGTGT \\
\hline RluD-up5' & TTCTTGCCACCAACTATTACGGC \\
\hline RluD-up3' & TTTGGGCCCAGCCTTATCCAAACGCAGAC \\
\hline RluD-down5' & TTTGGATCCAATTTAAAGCAGATATCCCAGAGA \\
\hline RluD-down3' & ATGTTGAAATATTGACAAAACCGTCT \\
\hline RluD-Cl & TCAGTATGCCAATCCTGAAG \\
\hline RluD-C2 & CTTGATACCACCAGTTCCC \\
\hline DprA-up5' & TTGTCCTATCTTGTGATTGTGCTC \\
\hline DprA-up3' & TTTGGGCCCTTCTACTCATCTATCTATTCGT \\
\hline DprA-down5' & TTTGGATCCAGTGGGCAAGATGTTCTTGC \\
\hline DprA-down3' & CGATAGAGGCGATAAGCATGGC \\
\hline DprA-Cl & GAGTGAAATCGAAGCCCTC \\
\hline DprA-C2 & AATCACGGGAGTCGAATCC \\
\hline HemK-up5' & CAACTACAAGTTGTAGAAGACCGT \\
\hline HemK-up3' & TTTGGGCCCTGAGCTAATTTCATTATTTGTTTA \\
\hline HemK-down5' & TTTGGATCCTTGGTCAAGATAGGATGGTTGT \\
\hline HemK-down3' & TCTCCTTTATTGTGTGACTAGTCC \\
\hline HemK-Cl & GAGAATGAAGTAGTAGCAATC \\
\hline HemK-C2 & CAGAGATCAGCATCATATGC \\
\hline HemK-pointl & CAACTCTTATTAAAAATTTGAAAATAATTGAGC (point mutations in bold) \\
\hline HemK-point2 & TTTCAAATTTTTAATAAGAGTTGATAAGACAAGGAG (point mutations in bold) \\
\hline YebC-up5' & TTTGCCACTAGTGCGTAAGCGG \\
\hline YebC-up3' & TTGGGCCCTACGTCCCATTAGGAATCTCC \\
\hline YebC-down5' & TTGGATCCACCTATAGAACATGATCCTAAGTG \\
\hline YebC-down3' & CTTCCCAATAGCCCAGATAGCC \\
\hline YebC-Cl & TTAGAGAGTGTACCGGGC \\
\hline YebC-C2 & AGGACTGTTTTGGAAAGGC \\
\hline ErmApa & TTTTTTGGGCCCTTCGTGTTCGTGCTGACTTGC \\
\hline ErmBam & TTTTTTGGATCCGATGTTGCTGATTAAGACGAGC \\
\hline SPI022-point I & TCTGTAGGCTAAACGACAGCTCAACCATTT (point mutations in bold) \\
\hline SPI022-point2 & AAATGGTTGAGCTGTCGTTTAGCCTACAGA (point mutations in bold) \\
\hline SPI022-up5' & TTTGCCACTAGTGCGTAAGCGG \\
\hline SPI022-up3' & TTGGGCCCTACGTCCCATTAGGAATCTCC \\
\hline SPI022-down5' & TTGGATCCACCTATAGAACATGATCCTAAGTG \\
\hline SPI022-down3' & CTTCCCAATAGCCCAGATAGCC \\
\hline $\mathrm{SPI} 1022-\mathrm{Cl}$ & TCAGATTGCTCAAGATGAGC \\
\hline SPI022-C2 & TTCCACCTGAAATAATACGGA \\
\hline SPI023-point I & GGTTGATGTCTCATATAGCTTACACATCAG (point mutations in bold) \\
\hline SPI023-point2 & CTGATGTGTAAGCTATATGAGACATCAACC (point mutations in bold) \\
\hline SPI023-up5' & AGATTGGTCAGTGACGGCA \\
\hline SPI023-up3' & TTGGGCCCAGTTTCTCTTAAAATCTCTTAGC \\
\hline SPI023-down5' & TTGGATCCAGAAACGGTTTATTCGCATCT \\
\hline SPI023-down3' & TTCCACCTGAAATAATACGGA \\
\hline SPI023-CI & TAGTGGAGCTATTGCTCTC \\
\hline SPI023-C2 & ATCCAACAAGTTTTGAGCAAC \\
\hline
\end{tabular}

\section{Availability and requirements}

NCBI: http://www.ncbi.nlm.nih.gov

R Project for Statistical Computing: http://www.rproject.org

\section{Authors' contributions}

JF and CB carried out the laboratory experiments. JF, CB, EM, HWW, SN and BHN designed the study and wrote the manuscript. All authors read and approved the final manuscript.

\section{Appendix}

See Table 3.

\section{Acknowledgements}

We thank Don Morrison for generously providing the Janus cassette for this work. Ingrid Andersson, Gunnel Möllerberg and Christina Johansson are also greatly acknowledged for excellent technical assistance. This work was supported by grants from the VINNOVA, Swedish Foundation for Strategic Research (SSF), Swedish Research Council, the EU programme PREVIS within the $6^{\text {th }}$ Framework Programme, Torsten and Ragnar Söderbergs foundation, and the Swedish Royal Academy of Sciences.

\section{References}

I. Carapetis JR, Steer AC, Mulholland EK, Weber M: The global burden of group A streptococcal diseases. Lancet Infect Dis 2005, 5(II):685-694 
2. Henrichsen J: Six newly recognized types of Streptococcus pneumoniae. J Clin Microbiol 1995, 33(10):2759-2762.

3. McCullers JA, Tuomanen El: Molecular pathogenesis of pneumococcal pneumonia. Front Biosci 200I, 6:D877-889.

4. Ripley-Petzoldt ML, Giebink GS, Juhn SK, Aeppli D, Tomasz A, Tuomanen $E$ : The contribution of pneumococcal cell wall to the pathogenesis of experimental otitis media. J Infect Dis 1988, I 57(2):245-255.

5. Barocchi MA, Ries J, Zogaj X, Hemsley C, Albiger B, Kanth A, Dahlberg S, Fernebro J, Moschioni M, Masignani V, et al.: A pneumococcal pilus influences virulence and host inflammatory responses. Proc Natl Acad Sci USA 2006, I03(8):2857-2862.

6. Hava DL, Camilli A: Large-scale identification of serotype 4 Streptococcus pneumoniae virulence factors. Mol Microbiol 2002, 45(5): I 389-| 406

7. Lau GW, Haataja S, Lonetto M, Kensit SE, Marra A, Bryant AP, McDevitt $D$, Morrison DA, Holden DW: A functional genomic analysis of type 3 Streptococcus pneumoniae virulence. Mol Microbiol 200I, 40(3):555-57I.

8. Polissi A, Pontiggia A, Feger G, Altieri M, Mottl H, Ferrari L, Simon D Large-scale identification of virulence genes from Streptococcus pneumoniae. Infect Immun 1998, 66(I 2):5620-5629.

9. De Lencastre $\mathrm{H}$, Tomasz A: From ecological reservoir to disease: the nasopharynx, day-care centres and drug-resistant clones of Streptococcus pneumoniae. J Antimicrob Chemother 2002, 50(Suppl S2):75-81.

10. Sandgren A, Sjostrom K, Olsson-Liljequist B, Christensson B, Samuelsson A, Kronvall G, Henriques Normark B: Effect of clonal and serotype-specific properties on the invasive capacity of Streptococcus pneumoniae. J Infect Dis 2004, I 89(5):785-796.

II. Brueggemann AB, Griffiths DT, Meats E, Peto T, Crook DW, Spratt BG: Clonal relationships between invasive and carriage Streptococcus pneumoniae and serotype- and clone-specific differences in invasive disease potential. J Infect Dis 2003, | 87(9): | 424- | 432.

12. Sandgren A, Albiger B, Orihuela CJ, Tuomanen E, Normark S, Henriques-Normark B: Virulence in mice of pneumococcal clonal types with known invasive disease potential in humans. J Infect Dis 2005, 192(5):79|-800.

13. Sjostrom K, Spindler C, Ortqvist A, Kalin M, Sandgren A, KuhlmannBerenzon S, Henriques-Normark B: Clonal and capsular types decide whether pneumococci will act as a primary or opportunistic pathogen. Clin Infect Dis 2006, 42(4):45I-459.

14. Garbom S, Forsberg A, Wolf-Watz H, Kihlberg BM: Identification of novel virulence-associated genes via genome analysis of hypothetical genes. Infect Immun 2004, 72(3): I333-1340.

15. Hoskins J, Alborn WE Jr, Arnold J, Blaszczak LC, Burgett S, DeHoff BS, Estrem ST, Fritz L, Fu DJ, Fuller W, et al:: Genome of the bacterium Streptococcus pneumoniae strain R6. J Bacteriol 200I, I 83(19):5709-57|7.

16. Tettelin H, Nelson KE, Paulsen IT, Eisen JA, Read TD, Peterson S, Heidelberg J, DeBoy RT, Haft DH, Dodson RJ, et al.: Complete genome sequence of a virulent isolate of Streptococcus pneumoniae. Science 200I, 293(5529):498-506.

17. Bruckner R, Nuhn M, Reichmann P, Weber B, Hakenbeck R: Mosaic genes and mosaic chromosomes-genomic variation in Streptococcus pneumoniae. Int J Med Microbiol 2004, 294(23): $157-168$.

18. Hiller NL, Janto B, Hogg JS, Boissy R, Yu S, Powell E, Keefe R, Ehrlich NE, Shen K, Hayes J, et al: Comparative genomic analyses of seventeen Streptococcus pneumoniae strains: insights into the pneumococcal supragenome. J Bacteriol 2007, 189(22):8186-8195.

19. Burnaugh AM, Frantz LI, King S): Growth of Streptococcus pneumoniae on human glycoconjugates is dependent upon the sequential activity of bacterial exoglycosidases. I Bacteriol 2008, I 90(I):22I-230.

20. Caldon $C E$, Yoong P, March PE: Evolution of a molecular switch: universal bacterial GTPases regulate ribosome function. Mol Microbiol 200I, 4I(2):289-297.

21. Teplyakov A, Obmolova G, Chu SY, Toedt J, Eisenstein E, Howard AJ, Gilliland GL: Crystal structure of the YchF protein reveals binding sites for GTP and nucleic acid. J Bacteriol 2003, | 85( I 4):403 I-4037.

22. Raychaudhuri S, Conrad J, Hall BG, Ofengand J: A pseudouridine synthase required for the formation of two universally con- served pseudouridines in ribosomal RNA is essential for normal growth of Escherichia coli. Rna 1998, 4(I I): I407-I4I7.

23. Ando T, Israel DA, Kusugami K, Blaser MJ: HP a member of the dprA family, is involved in natural transformation in Helicobacter pylori. J Bacteriol 0333, I 8 I (18):5572-5580.

24. Ogura M, Yamaguchi H, Kobayashi K, Ogasawara N, Fujita Y, Tanaka $\mathrm{T}$ : Whole-genome analysis of genes regulated by the Bacillus subtilis competence transcription factor ComK. I Bacteriol 2002, I 84(9):2344-235I.

25. Campbell EA, Choi SY, Masure HR: A competence regulon in Streptococcus pneumoniae revealed by genomic analysis. Mol Microbiol 1998, 27(5):929-939.

26. Sun YH, Bakshi S, Chalmers R, Tang CM: Functional genomics of Neisseria meningitidis pathogenesis. Nat Med 2000, 6(I I):1269-1273.

27. Heurgue-Hamard V, Champ S, Engstrom A, Ehrenberg M, Buckingham RH: The hemK gene in Escherichia coli encodes the N(5)glutamine methyltransferase that modifies peptide release factors. Embo J 2002, 2I(4):769-778.

28. Song JH, Ko KS, Lee JY, Baek JY, Oh WS, Yoon HS, Jeong JY, Chun J: Identification of essential genes in Streptococcus pneumoniae by allelic replacement mutagenesis. Mol Cells 2005, 19(3):365-374.

29. Thanassi JA, Hartman-Neumann SL, Dougherty TJ, Dougherty BA, Pucci MJ: Identification of I I 3 conserved essential genes using a high-throughput gene disruption system in Streptococcus pneumoniae. Nucleic Acids Res 2002, 30(14):3152-3162.

30. Berry AM, Yother J, Briles DE, Hansman D, Paton JC: Reduced virulence of a defined pneumolysin-negative mutant of Streptococcus pneumoniae. Infect Immun 1989, 57(7):2037-2042.

3I. Walker JA, Allen RL, Falmagne P, Johnson MK, Boulnois G]: Molecular cloning, characterization, and complete nucleotide sequence of the gene for pneumolysin, the sulfhydryl-activated toxin of Streptococcus pneumoniae. Infect Immun I987, 55(5): I I84-II89.

32. Marra A, Asundi J, Bartilson M, Lawson S, Fang F, Christine J, Wiesner C, Brigham D, Schneider WP, Hromockyj AE: Differential fluorescence induction analysis of Streptococcus pneumoniae identifies genes involved in pathogenesis. Infect Immun 2002, 70(3): $1422-1433$

33. Orihuela CJ, Radin JN, Sublett JE, Gao G, Kaushal D, Tuomanen El: Microarray analysis of pneumococcal gene expression during invasive disease. Infect Immun 2004, 72(10):5582-5596.

34. Bjorkman J, Hughes D, Andersson DI: Virulence of antibioticresistant Salmonella typhimurium. Proc Natl Acad Sci USA 1998, 95(7):3949-3953

35. Kaczanowska M, Ryden-Aulin M: The YrdC protein - a putative ribosome maturation factor. Biochim Biophys Acta 2005, 1727(2):87-96.

36. Comartin DJ, Brown ED: Non-ribosomal factors in ribosome subunit assembly are emerging targets for new antibacterial drugs. Curr Opin Pharmacol 2006, 6(5):453-458.

37. Giefing C, Meinke AL, Hanner M, Henics T, Bui MD, Gelbmann D, Lundberg U, Senn BM, Schunn M, Habel A, et al:: Discovery of a novel class of highly conserved vaccine antigens using genomic scale antigenic fingerprinting of pneumococcus with human antibodies. J Exp Med 2008, 205(I): ||7-|3|.

38. Lacks S, Hotchkiss RD: A study of the genetic material determining an enzyme activity in Pneumococcus. Acta Biochem Biophys Acad Sci Hung 1960, 39:508-517.

39. Sjostrom K, Blomberg C, Fernebro J, Dagerhamn J, Morfeldt E, Barocchi M, Browall S, Moschioni M, Andersson M, Henriques F, et al: Clonal success of piliated penicillin non-susceptible pneumococci. Proc Natl Acad Sci USA 2007, 104: 12907- 12.

40. Smyth GK: Linear models and empirical bayes methods for assessing differential expression in microarray experiments. Stat Appl Genet Mol Biol 2004, 3:Article3.

4I. Macrina FL, Tobian JA, Jones KR, Evans RP, Clewell DB: A cloning vector able to replicate in Escherichia coli and Streptococcus sanguis. Gene 1982, 19(3):345-353.

42. Pozzi G, Masala L, lannelli F, Manganelli R, Havarstein LS, Piccoli L, Simon D, Morrison DA: Competence for genetic transformation in encapsulated strains of Streptococcus pneumoniae: two allelic variants of the peptide pheromone. J Bacteriol 1996, I 78(20):6087-6090. 
43. Sung CK, Li H, Claverys JP, Morrison DA: An rpsL cassette, janus, for gene replacement through negative selection in Streptococcus pneumoniae. Appl Environ Microbiol 200I, 67(II):5190-5196.

44. Martin-Galiano AJ, de la Campa AG: High-efficiency generation of antibiotic-resistant strains of Streptococcus pneumoniae by PCR and transformation. Antimicrob Agents Chemother 2003, 47(4): $|257-126|$.

Publish with Bio Med Central and every scientist can read your work free of charge

"BioMed Central will be the most significant development for disseminating the results of biomedical research in our lifetime. " Sir Paul Nurse, Cancer Research UK

Your research papers will be:

- available free of charge to the entire biomedical community

- peer reviewed and published immediately upon acceptance

- cited in PubMed and archived on PubMed Central

- yours - you keep the copyright

Submit your manuscript here:

http://www.biomedcentral.com/info/publishing_adv.asp
BioMedcentral 